\title{
Simulation Study on Acquisition Process of Locomotion by using an Infant Robot
}

\author{
Katsuyoshi Tsujita and Tatsuya Masuda \\ Dept. of Electrical and Electronic Systems Engineering, Osaka Institute of Technology \\ Japan
}

\section{Introduction}

Locomotion is one of the basic functions of a mobile robot. Using legs is one of the strategies for accomplishing locomotion. The strategy allows a robot to move over rough terrain. Therefore, a considerable amount of research has been conducted on motion control of legged locomotion robots. This chapter treats the motion generation of an infant robot, with emphasis on the emergence of crawling locomotion.

In the future, a walking robot that can carry out various tasks on unstructured terrain will be required. The walking robot is required to achieve real-time adaptability to a changing environment. However, the mechanism from which the adaptive motion pattern emerges is not clear.

Recent biological research and psychological research on acquisition of motion have made great contributions and have given crucial hints as to how to overcome such problems. During spontaneous motion, such as crawling or straight walking, a lot of joints and ठ․ muscles are organized into a collective unit to be controlled as if this unit had fewer degrees $\underset{\Phi}{0}$ of freedom, but at the same time to retain the necessary flexibility for a changing environment (Bernstein, 1967). Gesell pointed out the principles of motor development in 드 human infants (Gesell, 1946). According to that research, some developmental principles in $\frac{\tau}{D}$ the acquisition of ontogenetic activities can be observed. One is directional trends in the $\stackrel{ \pm}{.}$ acquisition of ontogenetic activities; others are functional asymmetry in ontogenetic activities and self-regulation in ontogenetic activities. In addition, various studies have been made on the acquisition of motion especially that of locomotion (Newell, 1990; Thelen et al.,1986,1987; ¿ Clark et al.,1988; Burnside, 1927; Adolph, 1997; Savelsbergh, 1993). Moreover, the O⿱ $)$ development of motions has been proposed as being a dynamic interaction between the Tु nervous and musculo-skeletal systems. Rhythmic motion is generated by a central pattern

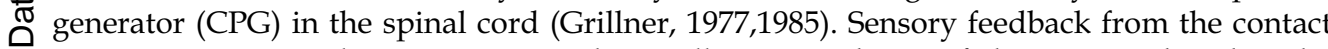
of sensors or joint angle sensors tunes the oscillation condition of the CPG and makes the D locomotion stable in limit cycle (Taga, 1991,1994). Furthermore, biological researches on 㲐 mode transition of the locomotion according to the situation or variance of the environment ᄃ are actively going on (Ijspeert, 2001). Based on these biological facts, research has been ळे conducted to clarify the mechanism for humans' acquisition of motion (Yamazaki, 1996; O Hase, 2002; Ni et al., 2003; Endo et al., 2004, Kuniyoshi et al., 2004). 
The knowledge acquired has inspired robotics researchers, and a considerable amount of research has been done on biologically inspired control systems for walking robots that are based on the CPG controller model, and that will enable them to adapt to variances in the environment (Fukuoka et al., 2003; Tsujita et al., 2001,2005; Lewis et al., 2001).

In this study, we propose a new control system for acquisition of the motion of an infant robot by using oscillators. The proposed control system consists of a spontaneous locomotion controller, reflex controller, and tones controller. The spontaneous locomotion controller is designed as follows. A nonlinear oscillator is assigned to each leg. The periodic trajectory of each leg is calculated as a function of the phase of its oscillator. Touch sensors at the tips of the legs are used to triggers dynamic interactions of the legs. The mutual entrainment of the oscillators generates an appropriate combination of phase differences according to the context of the changing environment, and this leads to a gait pattern. The network connections of the oscillators, specifically, the phase differences of the oscillators, are tuning parameters. These parameters are tuned according to a specific intension, i.e., an objective function for learning or optimization, such as stiffening joints or to moving forward at high energy efficiency. The reflex controller generates asymmetrical reflexes on antagonistic pairs of actuators. The idea of the architecture of tones control of the actuators are inspired by the biological studies. According to them, the tones are actively and adaptively controlled by the neuronal system in the 'basal ganglia' (Takakusaki, 2003). And this neuronal system stabilizes the posture, and obtains the stable locomotion by also controlling the oscillation of central pattern generator in the spinal cord. In this study, a type of tones controlling is considered as PD feedback control by adaptively changing the gains. The tones controller tunes the stiffness of the joints by changing the feedback gains adaptively according to the situations of the motion pattern of the system. These feedback gains are also refhed through learning or optimization.

We verified the effectiveness of the proposed control system with numerical simulations and hardware experiments.

\section{Framework of this study}

Fig.l summarizes the developmental acquisition of motion pattern in human infancy.

In this study, developmental acquisition is divided into four stages. In the first stage, the tones controller tunes the stiffness of the joints of the neck, trunk, hips, arms, and legs, in this order. In this stage, the intension of the infant is considered as that of making the controllable body system to perform a motion defhed by a command signal. In the second stage, primitive crawling locomotion emerges from using the alternative motion of hands by applying an asymmetry reflex to the hands' contact with the ground. In this stage, legs are not so skilled at generating propulsion force for locomotion. The intension of this stage is considered that of moving forward. In the third stage, adjustment of the tones of the legs' actuator is completed and the infant begins a perfect crawling locomotion that is fast with high energy-efficiency. The intension of this stage is considered as to move forward faster with less fatigue of actuators. The last stage is bipedal. The intension of this stage is considered as to raise the position of the eye higher.

In this chapter, these intensions are formulated as objective functions that are heuristically and dynamically controlled according to a particular developmental stage of the infant robot. The feedback gains of the actuators that govern tones (stiffness) of the joints and 
interaction strength among the oscillators are selected as tuning parameters for learning or optimization.

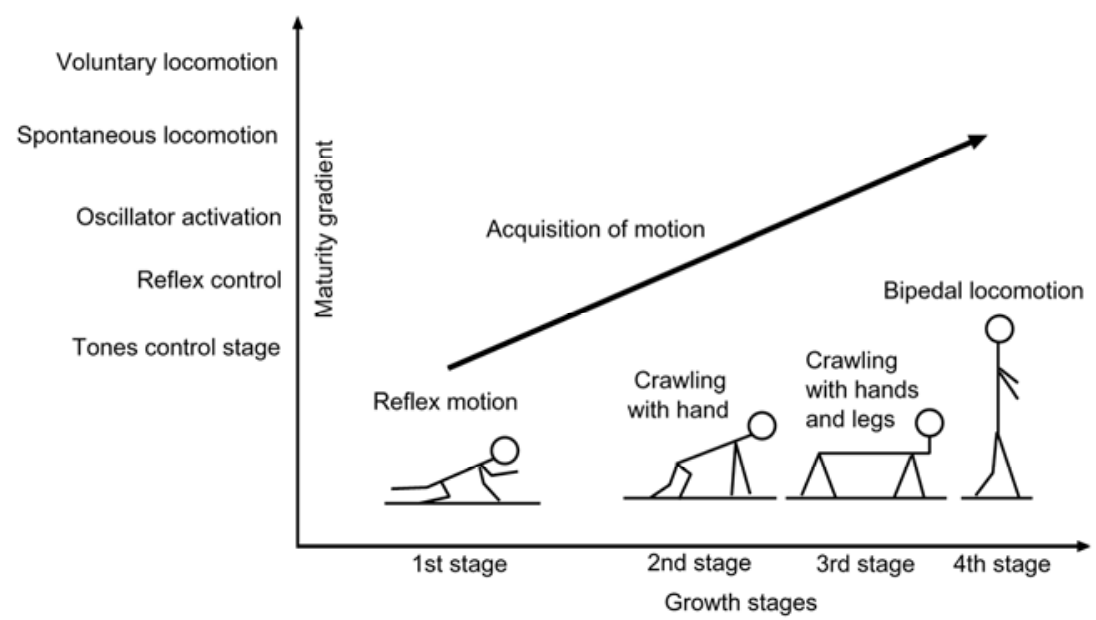

Figure 1. Growth cycle in acquisition of motions

\section{Model}

Fig. 2 is a schematic model of the infant robot. This robot has 28 DOF (Degrees of Freedom). The robot has a head, a torso, two arms, and two legs. Each arm consists of two links that are connected to each other through three degrees of freedom, (DOF) rotational joints at the shoulders and a one DOF rotational joint at the elbows. Each leg consists of three links that are connected to each other through rotational joints with three degrees of freedom (DOF) at the hip and ankle, and a one DOF rotational joint at the knee. The torso is composed of two parts, upper body and lower body. The upper body and the lower body are connected through a three DOF rotational joint. The arms and legs are connected to the torso with three DOF rotational joints. The head is connected to the torso with a three DOF rotational joint. Each subsystem is enumerated as 0: Upper body, 1:Head, 2:Lower body, 3:Left hand, 4:Right hand, 5:Left leg and 6:Right leg. We define $r_{k}^{(B)}$ and $\theta_{k}^{(0)}(k=1,2,3)$ as the components of position vector and Euler angle from inertial space to the coordinate system that is fixed on the upper body, respectively. The joint angle of the lower body to upper body is defined as $\theta^{(2)}$. We also defhe each joint angle of each corresponding joint, $\theta_{j}^{(i)}$ (i:subsystem no., $j$ : joint no.).

The state variable is defhed as follows;

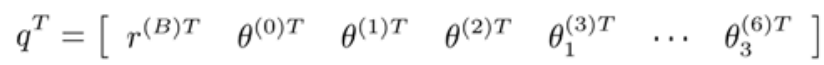

Equations of motion for state variable $q$ are derived using Lagrangian formulation as follows;

$$
M \ddot{q}+H(q, \dot{q})=G+T+\Lambda
$$


where $M$ is the generalized mass matrix and the term $M \ddot{q}$ expresses the inertia. The nonlinear term which includes Coriolis forces and centrifugal forces is $H(q, \dot{q})$, and $G$ is the gravity term. The component of $\boldsymbol{T}$ vector $T_{j}^{(i)}$ and $T^{(B)}$ are the input torque of the actuator at joint $j$ of leg $i$ and at the joint of torso. A is the reaction force from the ground at the point where the tip of the leg makes contact. We assume that friction exists between the ends of the legs and the ground. In this study, the candidates of the contact point of the robot body are guessed to be elbows, shoulders, knees, and four points at the rectangular corners of the feet.

The model of friction between the contact points of the ends of hands or legs and the ground is given as follows;

$$
\left\{\begin{aligned}
\Lambda_{j}^{i} & =-K_{E, \text { floor }} \Phi_{j}^{i}-K_{V, \text { floor }} \dot{\Phi}_{j}^{i} & & \left|\Lambda_{j}^{i}\right|<\mu \Lambda_{3}^{i} \\
\Lambda_{j}^{i} & =-\mu \Lambda_{3}^{i}-K_{V, \text { friction }} \dot{\Phi}_{j}^{i} & & \Lambda_{j}^{i}<-\mu \Lambda_{3}^{i} \\
\Lambda_{j}^{i} & =\mu \Lambda_{3}^{i}-K_{V, \text { floor }} \dot{\Phi}_{j}^{i} & & \Lambda_{j}^{i}>\mu \Lambda_{3}^{i} \\
& j=1,2 & &
\end{aligned}\right.
$$

where $\Lambda_{j, j}^{i}=1,2$ are horizontal friction force and $\Lambda_{3}^{i}$ is vertical reaction force at contact point i, respectively. $K_{E \text {,floor }}, K_{V \text {,floor }}, K_{V \text {, friction }}$ and $\mu$ are elastic coefficient and viscous coefficient of the visco-elastic model of the contact motion, the coefficient of viscous coefficient of friction of the floor, and the coefficient of stiction, respectively. The position of the end of hand or knee at contact point i is expressed as vector $\Phi_{j}^{i}$ in the inertial space with the component of $j=1,2,3$.

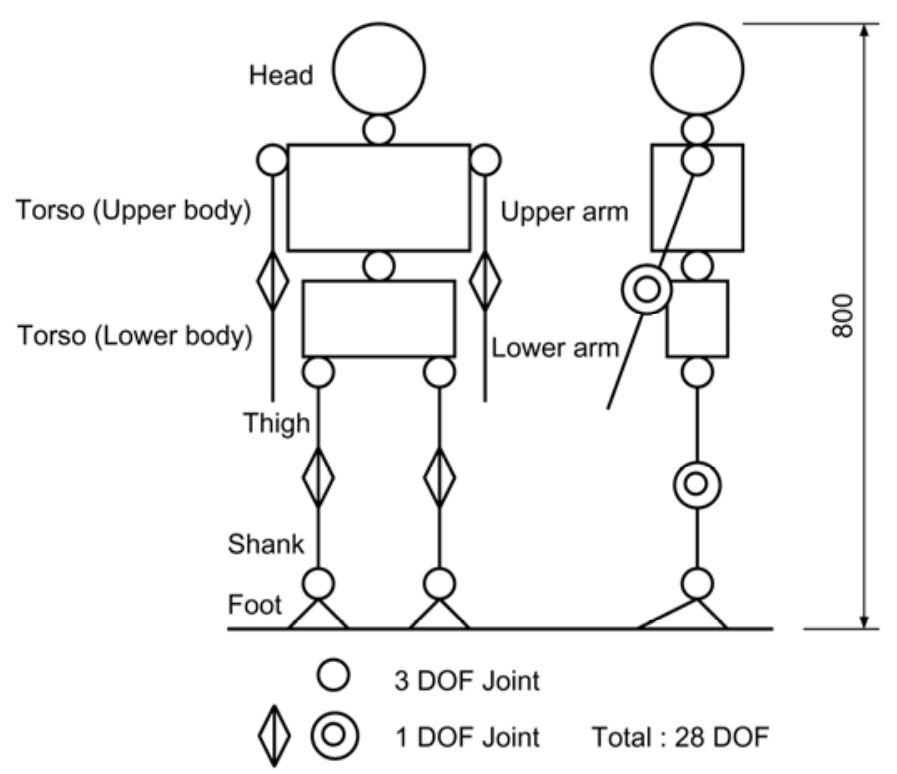

Figure 2. Schematic model of infant robot 


\section{Control System}

The architecture of the proposed control system is diagrammed in Fig. 3. The proposed control system has upward signal-flow and downward signal-flow. An oscillator for determining the rhythm and phase difference of periodic motions during locomotion is assigned for each leg, each hand, the torso, head, and a pace maker. The state of the oscillator for each part is expressed as follows;

$$
\begin{gathered}
z^{(i)}=\exp \left(j \phi^{(i)}\right) \\
i=3, \cdots, 6 \text { (subsystem no.) } \\
z^{(B)}=\exp \left(j \phi^{(B)}\right) \\
z_{\text {pace }}=\exp \left(j \phi_{\text {pace }}\right)
\end{gathered}
$$

where $\phi^{(i)}, \phi^{(B)}$ and $\phi_{\text {pace }}$ are the phase of oscillator $i$, torso and that of the pacemaker oscillator.

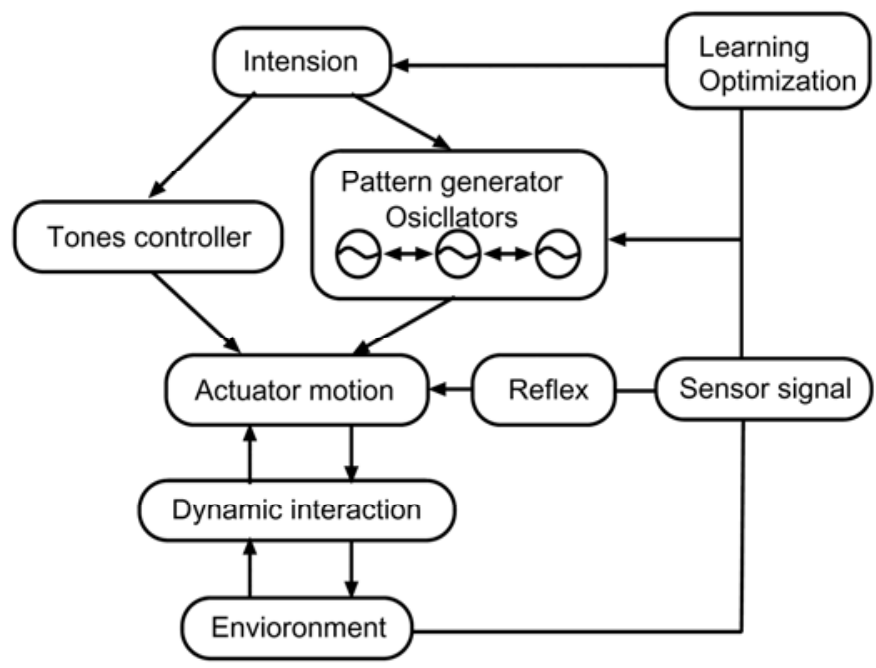

Figure 3. Control system

The pattern generator has the nonlinear oscillator network illustrated in Fig. 4, and it encodes the nominal trajectory of each joint angle of the hand and leg in terms of the phase of the oscillator that is given to the actuator inputs as a command signal. The tones controller tunes and varies the joint stiffness to correspond to the motion.

On the other hand, feedback signals of the contact sensors or attitude sensors are given to the reflex controller, and a pattern generator that is a learning or optimization process for the system. First, the contact sensor signal is input to the reflex controller as feedback signal. The reflex controller makes an immediate reflex to the contact signal or attitude signals to stabilize the posture of the torso. Then, the signal is also input to the pattern generator to reset the oscillator phase from the swinging stage to the supporting stage at the moment of 
contact, and vice versa. This dynamic interaction loop between pattern generator, environment, and actuator motion creates mutual entrainment and obtains a stable limit cycle for locomotion. Furthermore, the proposed system has a learning or optimization mechanism of using the feedback signal of the sensors. This system-tuning mechanism optimizes the parameters of the phase difference of the oscillators, actuator stiffness, and the amplitude of the synchronizing torso twisting motion.

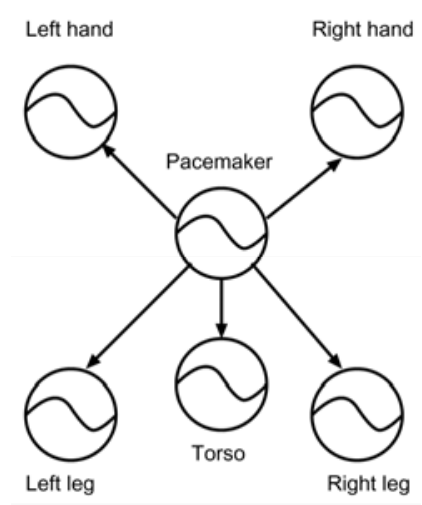

Figure 4. Oscillator network on pattern generator

The oscillator network in the pattern generator is formulated as follows;

$$
\begin{aligned}
\phi^{(i)} & =\phi_{\text {pace }}+\gamma_{j}^{(i)} \\
\phi^{(B)} & =\phi_{\text {pace }}+\gamma^{(B)}
\end{aligned}
$$

where $\gamma^{(i)}, \gamma^{(B)}$ are the phase differences of oscillator $i$ and torso to the pacemaker.

The trajectories of the hands and legs $r_{j}^{(i)}(j$ : joint no.) are given as function of the oscillator phase as follows;

$$
r_{j}^{(i)}= \begin{cases}f_{s w}\left(\phi^{(i)}\right) & \text { Swing stage } \\ f_{s p}\left(\phi^{(i)}\right) & \text { Support stage }\end{cases}
$$

The motion of the torso is a periodic one as follows;

$$
\theta^{(2)}=A_{t} \sin \left(\phi^{(B)}\right)
$$

The desired angle of each joint is calculated as a function of oscillator phase as follows;

$$
\begin{gathered}
\hat{\theta}_{j}^{(i)}=\theta_{j}^{(i)}\left(r_{j}^{(i)}\left(\phi^{(i)}\right)\right) \\
i=3, \cdots, 6 \text { subsystem no. } j: \text { joint no. }
\end{gathered}
$$

The actuator inputs are designed as follows;

$$
T_{j}^{(i)}=K_{P j}^{(i)}\left(\hat{\theta}_{j}^{(i)}-\theta_{j}^{(i)}\right)-K_{D j}^{(i)} \dot{\theta}_{j}^{(i)}
$$




$$
T^{(B)}=K_{P B}\left(\hat{\theta}^{(2)}-\theta^{(2)}\right)-K_{D B} \dot{\theta}^{(2)}
$$

where $K_{P j}^{(i)}, K_{D j}^{(i)}, K_{P B}$ and $K_{D B}$ are feedback gains and these parameters are controlled as the tones of the actuators and are tuned in the learning process or optimization in the developmental process.

\subsection{Optimization}

The parameters in the control system are tuned to generate motion patterns such as crawling locomotion. Optimization parameters are as follows;

$$
C=\left[K_{P j}^{(i)}, K_{D j}^{(i)}, K_{P B}, K_{D B}, \gamma^{(i)}, \gamma^{(B)}, A_{t}\right]
$$

The objective function $\boldsymbol{U}$ is given as follows;

$$
\begin{gathered}
U=\alpha \int_{0}^{t} \sum_{i, j}\left(\theta_{j}^{(i)}-\hat{\theta}_{j}^{(i)}\right) d t+\beta \int_{0}^{t}\left\|r^{(B)}\right\| d t+w P \\
P=\int_{0}^{t} \sum_{i, j} T_{j}^{(i) T} \dot{\theta}_{j}^{(i)} d t
\end{gathered}
$$

where $w$ is weight parameter, $\boldsymbol{a}$ and $\boldsymbol{\beta}$ are intension parameters which makes orientation of the development. These parameters are heuristically controlled as time sequence as follows;

$$
\alpha+\beta=w
$$

$$
\begin{gathered}
\alpha \gg \beta \text { early stage on development } \\
\alpha \sim \beta \text { middle stage on development } \\
\alpha \ll \beta \text { last stage on development }
\end{gathered}
$$

In this study, for simplicity of the problem, simulated annealing (SA) method is adopted for the optimization method.

\section{Numerical Simulation}

We implemented numerical simulations to verify the effectiveness of the proposed control system. Table 1 lists the physical parameters of the robot that are used in numerical simulations.

The nominal time period of the swinging stage was chosen as 0.25 [sec]. First, we investigated the tuning properties of the feedback gains. Figures 5 and 6 are optimization profiles ( i.e., training profiles) of feedback gains $K_{P j}^{(i)}$ and $K_{D j}^{(i)}$, respectively. The feedback gains were well tuned and converged to appropriate values, and these gains made the joints of the body, hands, and legs adequately stiff to control the motions effectively. 


\begin{tabular}{|lc|lc|}
\hline Segment length & {$[\mathrm{m}]$} & Segment mass & {$[\mathrm{kg}]$} \\
\hline Head & 0.16 & Head & 1.0 \\
Torso & 0.35 & Torso & 6.2 \\
Upper arm & 0.12 & Upper arm & 0.19 \\
Lower arm & 0.14 & Lower arm & 0.20 \\
Thigh & 0.11 & Thigh & 0.41 \\
Shank & 0.12 & Shank & 0.21 \\
Foot & 0.06 & Foot & 0.12 \\
\hline
\end{tabular}

Table 1. Physical parameters

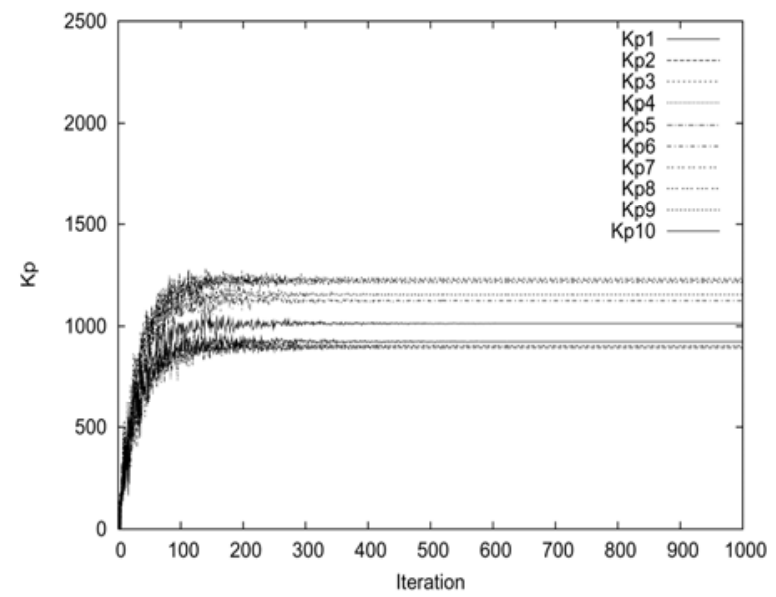

Figure 5. Tuned feedback gains $\left(K_{P}\right)$

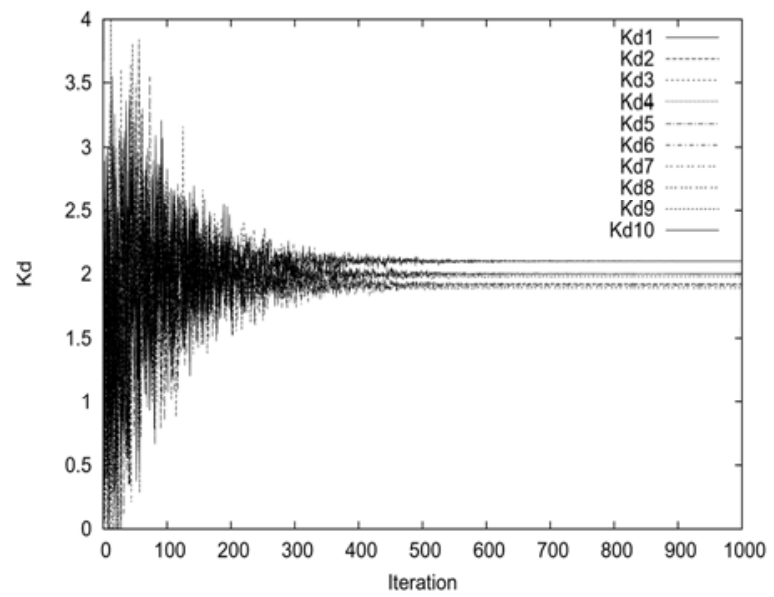

Figure 6. Tuned feedback gains $\left(K_{D}\right)$ 
Fig. 7 shows the training properties of the phase differences of the oscillator network in the pattern generator. We found that the converged values indicated the locomotion pattern was a diagonal sequence, i.e., left hand and right leg were in phase, right hand and left leg were also in phase, while left and right hands or legs were anti-phase. This phase difference made an effective crawling locomotion and the locomotion was smooth.

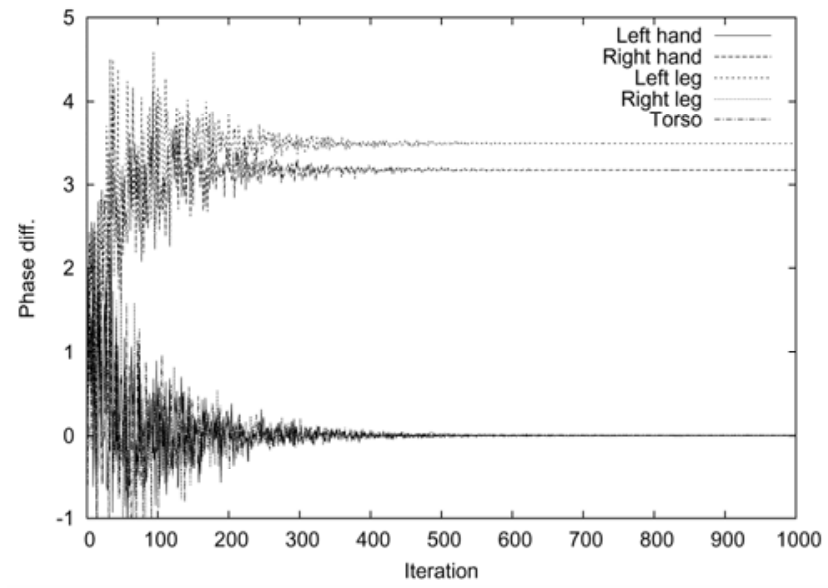

Figure 7. Phase differences of oscillators

Figures 8 and 9 are roll motion of the torso (i.e. roll angle and roll angular velocity in the phase plane). Note that the range of the fgures are not same. The result shows that the well trained system obtained stable and steady locomotion and form a limit cycle. However, in the other case (not trained), there is no limit cycle nor attractor.

Fig. 10 displays the simulation result of the crawling locomotion of the infant robot when it was well trained on its developmental parameters. In this case, the robot used a twisting motion of its torso and achieved a stable and low-energy-cost crawling locomotion. This result implies that the feedback signal of the contact sensor created mutual entrainment of the oscillators, body, and environment, and it achieved a stable limit cycle. Moreover, the appropriate phase difference for the hands, torso, and legs was obtained through the developmental training, i.e., the learning or optimization of control parameters. On the other hand, Fig. 11 shows snapshots of the crawling motion of the untrained robot. We found that in this case the robot lost its stable posture during locomotion. This result was due inappropriate phase differences of the oscillators, and too much actuator stiffness.

Fig. 12 shows the vertical reaction force at the contact point between the leg and the ground. This is for the well-trained robot. We found that the robot had a constant walking cycle and a limited cycle of crawling locomotion. On the other hand, Fig. 13 shows the case of the untrained robot. In this case, the time period from one contact moment to the next contact moment, i.e., the walking cycle, was not constant, but fluctuated. This means the robot had no stable limit cycle in crawling locomotion. Indeed, the robot could not continue stable locomotion and finally fell down.

From these fgures, one can see that the robot with the proposed control system could be well trained, could obtain appropriate actuator stiffness and locomotion conditions, and could autonomously achieve stable crawling locomotion. 
The effectiveness of the proposed control system was verified by these results.

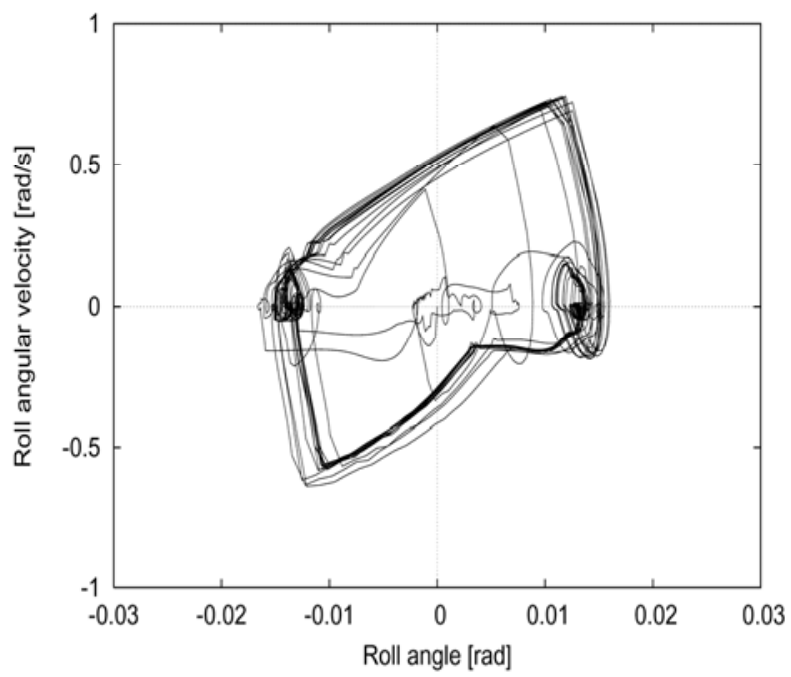

Figure 8. Roll angle and angular velocity (well trained)

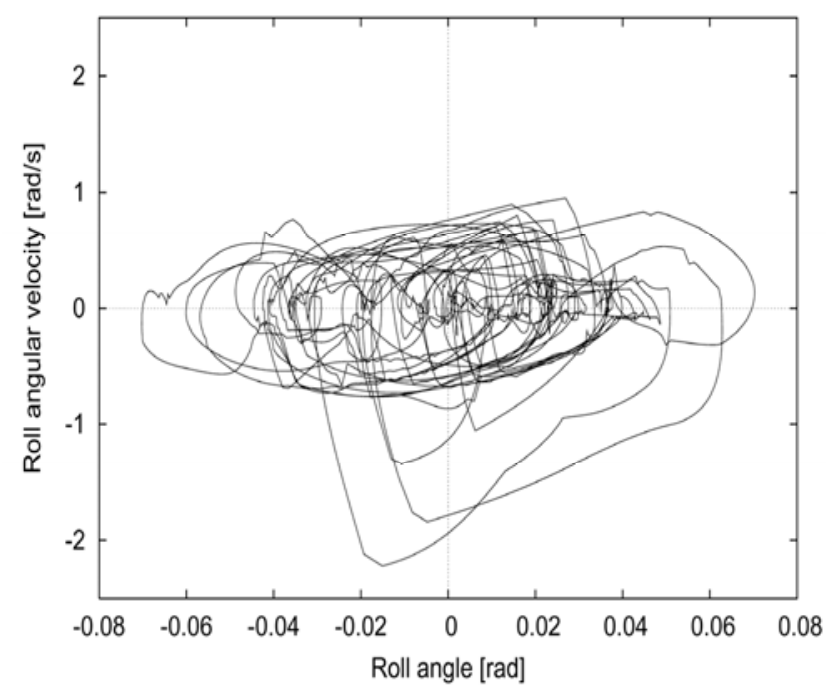

Figure 9. Roll angle and angular velocity (not trained)

Furthermore, we may note that the order of intension parameter dominance, that is, the dynamics of the constraint conditions for learning or optimization, is very important for motion acquisition. Indeed, when the order of intension parameter dominance for tuning the controller was changed from that of Eqs. (20) - (23), we could not obtain the crawling locomotion or appropriate joint stiffness, at least within a practical simulation time. 


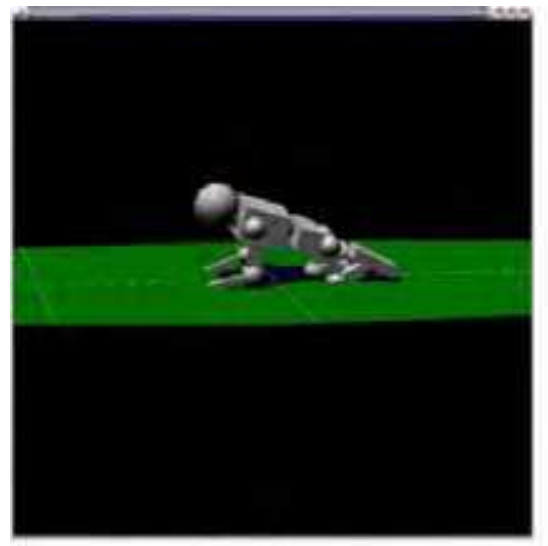

(1)

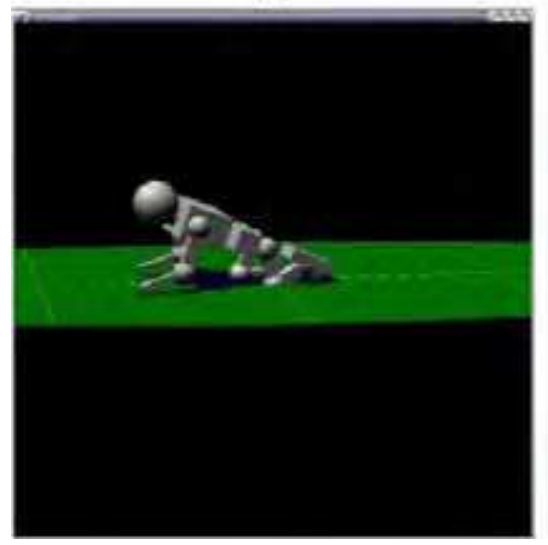

Figure 10. Snapshot taken during locomotion (well trained)
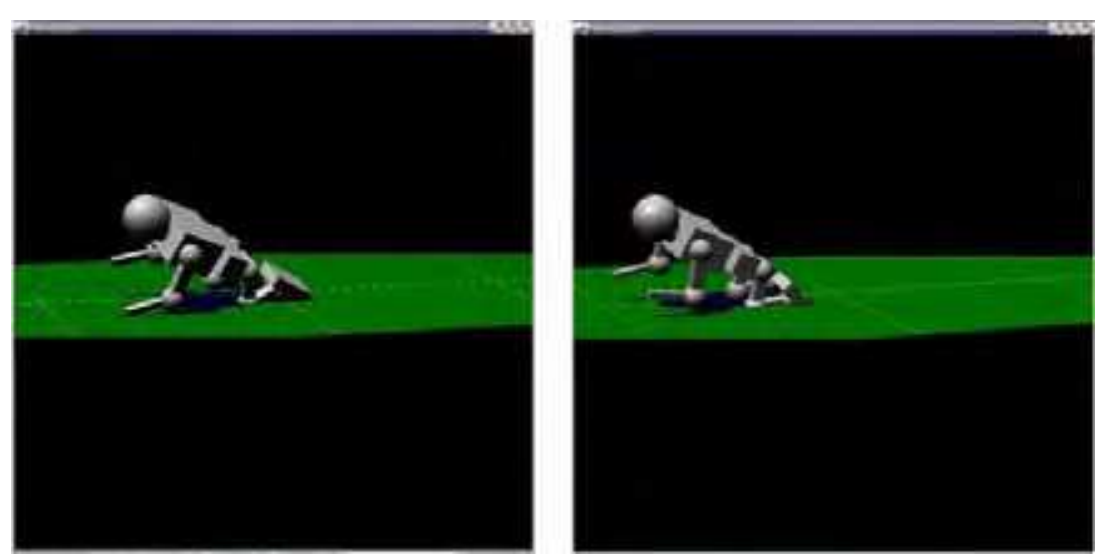

Figure 1l. Snapshot taken during locomotion (untrained)

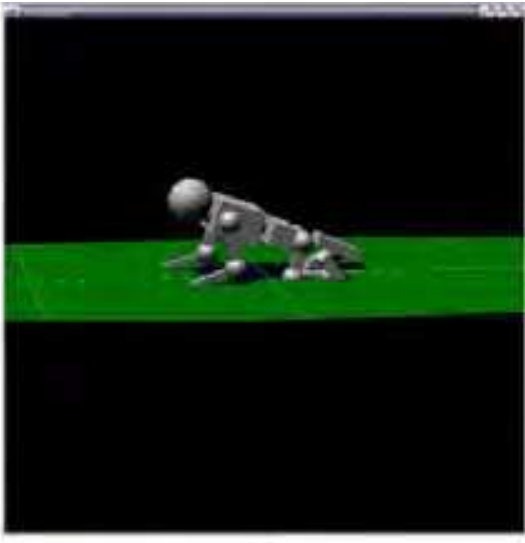

(2)

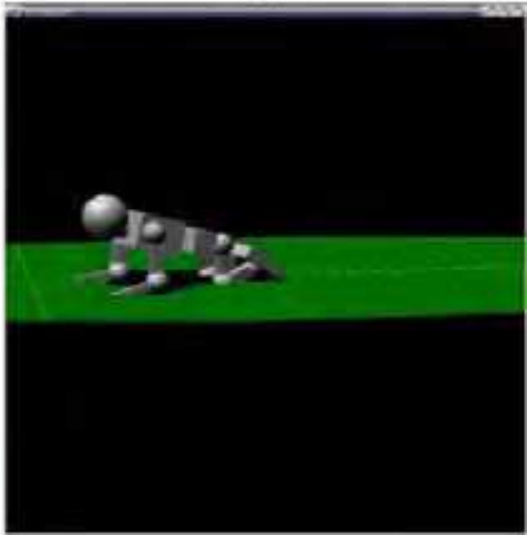




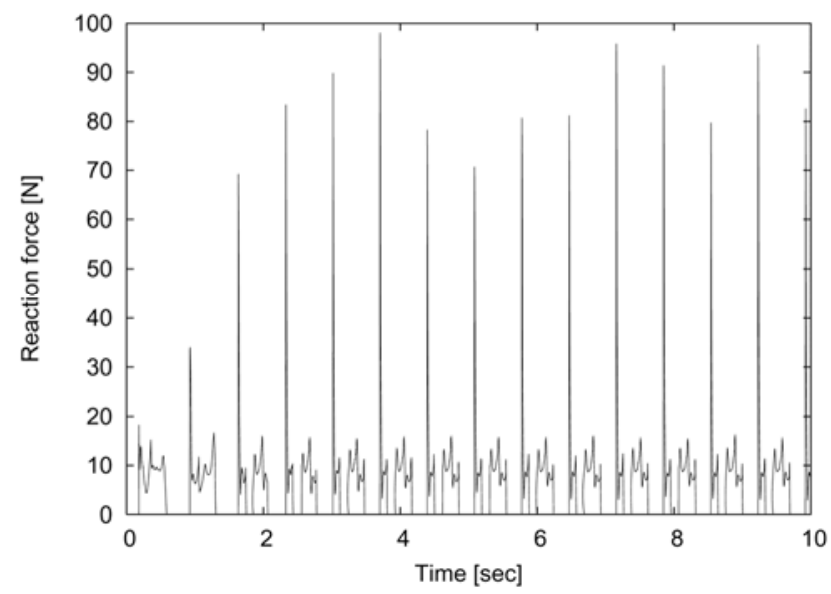

Figure 12. Reaction force on the leg (well trained)

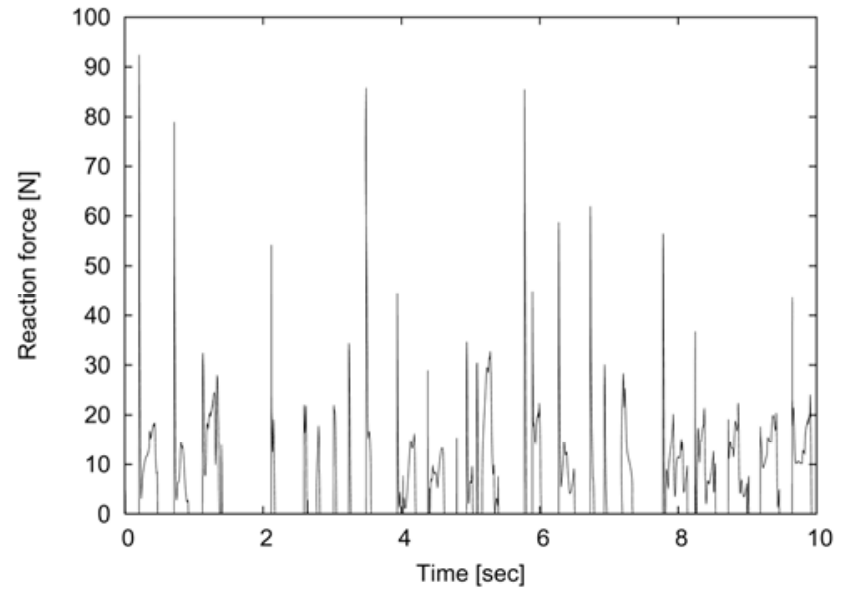

Figure 13. Reaction force on the leg (not trained)

\section{Conclusion}

This chapter presented a simulation method for simulating, and thereby clarifying, the development process of human locomotion that uses a model of an infant robot. The infant robot has many degrees of freedom. By making constraints and coordination for many DOF of using "intensions" such as "want to stay sitting," or "want to move more forward," etc., various motion patterns emerge according to a specific intension. In this study, this type of intension is given as the objective function and development of motion pattern, and is simulated as an optimization or learning problem. Dynamically changing the intension according to the development process changed the motion pattern of the infant robot, and it developed from sitting to crawling, toward locomotion. We investigated the process of this type of development in the infant robot with numerical simulations. 


\section{Acknowledgment}

This work has been partially supported by a Grant-in-Aid for Scientific Research on Priority Areas "Emergence of Adaptive Motor Function through Interaction between Body, Brain and Environment" from the Japanese Ministry of Education, Culture, Sports, Science and Technology.

\section{References}

Berstein, N. (1967) Co-ordination and regulation of movements, Oxford, Pergamon press, New York Gesell,A. (1946). The ontogenesis of infant behavior, The ontogenesis of infant behavior, edited by L. Carmichael, Wiley, New York, pp. 295-331

Newell, K \& Emmerik, R. (1990) Are Gesell's developmental principles general principles for the acquisition of coordination? Advances in motor development research, Vol.3, pp.143-164

Burnside, L. (1927). Coordination in the locomotion of infants, Genetic psychology monographs, Vol.2, No.5, pp.283-341

Thelen, E, Skala,K \& Kelso,J. (1987). The Dynamic Nature of Early Coordination: Evidence From Bilateral Leg Movements in Young Infants, Developmental Psychology, Vol.23, No.2, pp. 179-186

Thelen, E. (1986). Treadmill-elicited Stepping in Seven-Monthh-Old Infants, Child Development, 57, pp. 1498-1506

Clark, J Whitall, J \& Phillips, S. (1988). Human interlimb coordination: The first 6 months of independent walking, Developmental Psychobiology, Vol.21, 5, pp.445-456

Burnside, L. (1927). Coordination in the Locomotion of Infants, Genetic Psychology Monographs, Vol.2, No.5, pp.285-372

Adolph, K. (1997). Learning in the Development of Infant Locomotion, Monographs of the Society for Research in Child Development, Vol.62, No.3

Savelsbergh, G. (1993). The Development of Coordination in Infancy, Advances in Psychology, No.97

Grillner, S. (1977). Locomotion in vertebrates:Central mechanisms and reflex interaction, Physiological Review, No.55, pp.367-371

Grillner, S. (1985). Neurobiological bases of rhythmic motor acts in vertebrates, Science, Vol.228, pp.143-149

Taga, G, Yamaguchi, Y \& Shimizu, H. (1991). Self-organized control of bipedal locomotion by neural oscillators in unpredictable environment, Biological Cybernetics, No.65, pp.147-159

Taga, G. (1994). Emergence of bipedal locomotion through entrainment among the neuromusculo-skeletal system and the environment, Physica D, No.75, pp.190-208

Ijspeert, A. (2001). A connectionist central pattern generator for the atuatic and terrestrial gaits of a simulated salamander, Biological Cybernetics,

Yamazaki, N, Hase, K, Ogihara, N \& Hayamizu, N. (1996). Biomechanical Analysis of the Development of Human Bipedal Walking by a Neuro-Musculo-Skeletal Model, Folia Primatologica, Vol.66, pp.253-271

Hase, K., Yamazaki, N. (2002). Computer simulation study of human locomotion with a three-dimensional entire-body neuro-musculo-skeletal model. I. Acquisition of normal walking, JSME International Journal, Series C, 45, 4, pp. 1040-1050 
Hase, K., Obinata, G. (2002). Computer simulation study of human locomotion with a threedimensional entire-body neuro-musculo-skeletal model. II. Biomechanical relationship between walking stability and neuro-musculo-skeletal system, JSME International Journal, Series C, 45, 4, pp.1051-1057

Hase, K., Obuchi, S. (2002). Computer simulation study of human locomotion with a threedimensional entire-body neuro-musculo-skeletal model. III. Simulation of pathological walking and its application to rehabilitation engineering, JSME International Journal, Series C, 45, 4, pp.1058-1064

Hase, K., Yokoi, T. (2002). Computer simulation study of human locomotion with a threedimensional entire-body neuro-musculo-skeletal model. IV. Simulation of running motion and its transition process, JSME International Journal, Series C, 45, 4, pp.1065-1072

Ni, J, Hiramatsu,S \& Kato,A. (2003). A Model of Neuro-Musculo-Skeletal System for Human Locomotion Under Position Constraint Condition, Journal of Biomechanical Engineering, ASME, Vol.125, pp.499-506

G. Endo, G, Morimoto,J, Nakanishi,J \& Cheng,G. (2004). An Empirical Exploration of a Neural Oscillator for Bipedal Locomotion Control, Proc. of the 2004 IEEE Int. Conf. on Robotics and Automation, New Orleans, pp.3036-3042

Kuniyoshi, et al. (2004). From humanoid embodiment to theory of mind. In: Embodied artificial intelligence, lecture notes in Computer Science Series, Artificial Intelligence Subseries, F. lida, R. Pfeifer, L. Steels, \& Y. Kuniyoshi (Eds.), Vol. 3139, pp. 202-218, Springer, New York

Fukuoka, Y, Kimura,H, \& Cohen,A. (2003). Adaptive Dynamic Walking of a Quadruped Robot on Irregular Terrain Based on Biological Concepts, The International Journal of Robotics Research, 22, No. 3, pp.187-202

Tsujita, K, Tsuchiya,K \& Onat,A. (2001). Adaptive Gait Pattern Control of a Quadruped Locomotion Robot, Proc. IEEE/RSJ Int Conf on Intelligent Robots and Systems (IROS 2001), pp.2318-2325

Tsujita, K, H.Toui \& Tsuchiya,K. (2005). Dynamic Turning Control of a Quadruped Locomotion Robot using Oscillators, Advanced Robotics, Vol.19, No.10, pp.1115-133

Lewis, M, Hartmann,M, Etienne-cummings,R \& Cohen,A. (2001). Control of a robot leg with an adaptive a VLSI CPG chip, Neurocomp.,pp.1409-1421

Takakusaki, K, Habaguchi,T, Ohtinata-Sugimoto,J, Saitoh.K \& Sakamoto,T. (2003). Basal Ganglia Efferents to the Brainstem Centers Controlling Postural Muscle Tone and Locomotion: A New Concept for Understanding Motor Disorders in Basal Ganglia Dysfunction, Neuroscience, Vol.119, pp.293-308

Ogino, M, Katoh,Y, Aono,M, Asada,M, \& Hosoda,K. Reinforcement Learing of Humanoid Rhythmic Walking Parameters based on Visual Information,

Morimoto, J, Nakanishi, J, Endo,G, \& Cheng,G. (2005). Poincare-Map-Based Reinforcement Learning For Biped Walking, Proc. of the 2005 IEEE Int. Conf. on Robotics and Automation, Barcelona

STedrake, R \& Seung, H. (2002). Improved Dynamic Stability Using Reinforcement Learning, Proc. of CLAWAR '02

Bertenthal, B \& Bai, Dina. (1989). Infants' Sensitivity to Optical Flow for Controlling Posture, Developmental Psychology, Vol.25, No.6, pp.936-945 


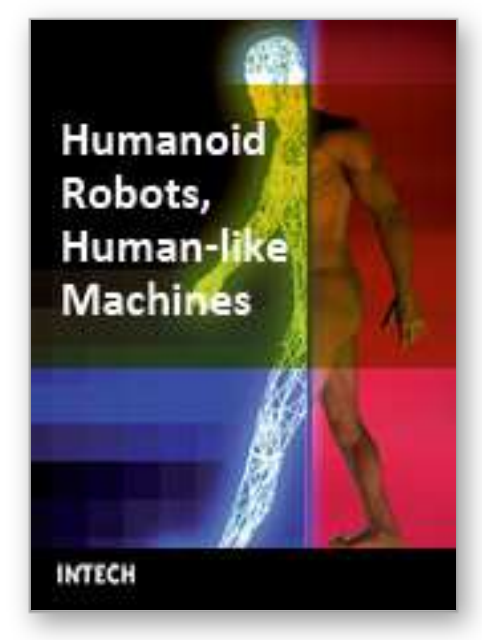

\author{
Humanoid Robots, Human-like Machines \\ Edited by Matthias Hackel
}

ISBN 978-3-902613-07-3

Hard cover, 642 pages

Publisher I-Tech Education and Publishing

Published online 01, June, 2007

Published in print edition June, 2007

In this book the variety of humanoid robotic research can be obtained. This book is divided in four parts: Hardware Development: Components and Systems, Biped Motion: Walking, Running and Self-orientation, Sensing the Environment: Acquisition, Data Processing and Control and Mind Organisation: Learning and Interaction. The first part of the book deals with remarkable hardware developments, whereby complete humanoid robotic systems are as well described as partial solutions. In the second part diverse results around the biped motion of humanoid robots are presented. The autonomous, efficient and adaptive two-legged walking is one of the main challenge in humanoid robotics. The two-legged walking will enable humanoid robots to enter our environment without rearrangement. Developments in the field of visual sensors, data acquisition, processing and control are to be observed in third part of the book. In the fourth part some "mind building" and communication technologies are presented.

\title{
How to reference
}

In order to correctly reference this scholarly work, feel free to copy and paste the following:

Katsuyoshi Tsujita and Tatsuya Masuda (2007). Simulation Study on Acquisition Process of Locomotion by Using an Infant Robot, Humanoid Robots, Human-like Machines, Matthias Hackel (Ed.), ISBN: 978-3-90261307-3, InTech, Available from:

http://www.intechopen.com/books/humanoid_robots_human_like_machines/simulation_study_on_acquisition_ process_of_locomotion_by_using_an_infant_robot

\section{INTECH}

open science | open minds

\author{
InTech Europe \\ University Campus STeP Ri \\ Slavka Krautzeka 83/A \\ 51000 Rijeka, Croatia \\ Phone: +385 (51) 770447 \\ Fax: +385 (51) 686166 \\ www.intechopen.com
}

\author{
InTech China \\ Unit 405, Office Block, Hotel Equatorial Shanghai \\ No.65, Yan An Road (West), Shanghai, 200040, China \\ 中国上海市延安西路65号上海国际贵都大饭店办公楼 405 单元 \\ Phone: +86-21-62489820 \\ Fax: +86-21-62489821
}


(C) 2007 The Author(s). Licensee IntechOpen. This chapter is distributed under the terms of the Creative Commons Attribution-NonCommercial-ShareAlike-3.0 License, which permits use, distribution and reproduction for non-commercial purposes, provided the original is properly cited and derivative works building on this content are distributed under the same license. 